\title{
Use of PET-CT Imaging to Identify Femoral-Popliteal Stent Infection
}

Angela M. Chen, MD ${ }^{1}$, Emily M. Serafin, MD ${ }^{1}$, and Raghu L. Motaganahalli, MD ${ }^{1}$

${ }^{1}$ Indiana University School of Medicine, Indianapolis, IN, USA

Corresponding Author: Raghu L. Motaganahalli, Indiana University School of Medicine, $1801 \mathrm{~N}$ Senate Blvd MPC-2 Suite 3500, Indianapolis, IN 46202, USA. Email: rmotagan@iupui.edu

A 58-year-old male was admitted with several months of a nonhealing sinus draining through the right popliteal fossa. He had a history of type 2 diabetes mellitus, former tobacco abuse. He had undergone repair of abdominal aortic aneurysm, besides repair of bilateral femoral artery aneurysm, and right popliteal artery aneurysm repair with Viabahn stent graft 7 years ago. Due to stent occlusion, subsequently, he later had right femoral to tibial artery bypass with vein.

Computed tomography (CT) angiogram showed a fluid col-lection in the popliteal fossa around the stent graft and concluded that there was not edema around the graft to suggest infection (Figure). He had patent vein bypass (arrowhead, Figure A). Bruggink et al1 showed that single Flurodeoxyglucose-Positron emission tomography (FDG-PET) had improved sensitivity and specificity for detecting vascular prosthetic graft infection compared to CT ( $93 \%$ and $70 \%$ vs 56\% and 57\%, respectively). When PET-CT was used for this patient, focal hyper metabolic activity was seen along the course of the right superficial femoral and popliteal stent graft (B), corresponding to peripherally enhancing fluid and/or soft tissue surrounding the stented vessel and extending into the posterior thigh soft tissues (B). The increased uptake on PET-CT was interpreted as acute infection or a developing abscess.

The femoropopliteal stent was explanted using combined medial and posterior approach. There was erosion of the vessel wall due to infection (C: exposure, D: explanted stent sent for culture). Entire segment of the stent from the mid superficial femoral to the popliteal artery was excised with drainage of the abscess cavity.

This is the author's manuscript of the work published in final form as:

Chen, A. M., Serafin, E. M., \& Motaganahalli, R. L. (2019). Use of PET-CT Imaging to Identify Femoral-Popliteal Stent Infection. Vascular and Endovascular Surgery. https://doi.org/10.1177/1538574419831479 
The patient was treated with intravenous vancomycin and ceftriaxone initially. Wound cultures did not grow any organ-isms, while the cultures from the explanted stent grew Streptococcus agalactiae. He was discharged on cefpodoxime and had no postprocedural complications during his 6-month follow-up. Informed consent has been obtained from the patient (or patient's guardian) for publication of the case report and accompanying images.

\section{Declaration of Conflicting Interests}

The author(s) declared no potential conflicts of interest with respect to the research, authorship, and/or publication of this article.

\section{Funding}

The author(s) received no financial support for the research, author-ship, and/or publication of this article.

\section{Reference}

1. Bruggink JL, Glaudemans AW, Saleem BR, et al. Accuracy of FDG-PET-CT in the diagnostic work-up of vascular prosthetic graft infection. Eur J Vasc Endovasc Surg. 2010;40(3): 348-354. 


\section{Figure}

Showing infected popliteal stent with increased uptake on FDG-PET scan correlated with intra operative findings of eroded popliteal artery due to infection.

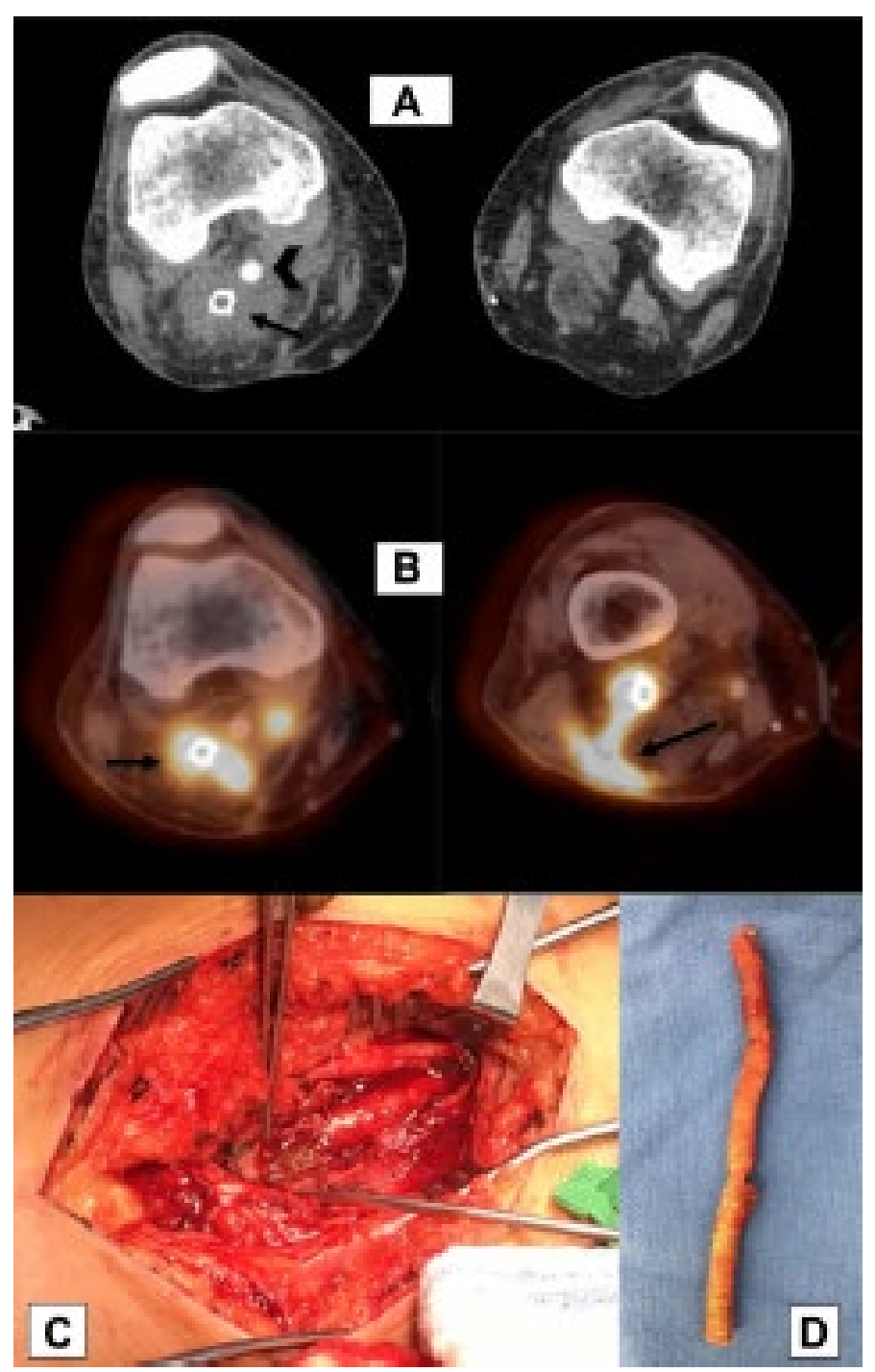

